Cahiers de Narratologie

Analyse et théorie narratives

L'Art du roman chez Umberto Eco

\title{
Baudolino, une fascination sémiotique pour le mensonge
}

Mohamed Bernoussi

\section{(2) OpenEdition}

12 Journals

\section{Electronic version}

URL: http://journals.openedition.org/narratologie/8216

DOI: 10.4000/narratologie.8216

ISSN: 1765-307X

\section{Publisher}

LIRCES

\section{Electronic reference}

Mohamed Bernoussi, «Baudolino, une fascination sémiotique pour le mensonge », Cahiers de Narratologie [Online], 33 | 2018, Online since 23 July 2018, connection on 15 November 2019. URL http://journals.openedition.org/narratologie/8216 ; DOI : 10.4000/narratologie.8216

This text was automatically generated on 15 November 2019.

Article L.111-1 du Code de la propriété intellectuelle. 


\title{
Baudolino, une fascination sémiotique pour le mensonge
}

\author{
Mohamed Bernoussi
}

1 Avant le roman éponyme, le personnage de Baudolino est évoqué dans Comment voyager avec un saumon. Eco le présente comme figure typique et indispensable lorsqu'il faut parler de sa ville natale Alexandria. Dans le roman éponyme, Baudolino conserve son ancrage régionaliste, mais se distingue surtout comme figure emblématique du mensonge, principalement le reliquaire.

2 Nous sommes au XIème siècle, de nouveau au Moyen Age, Baudolino rencontre Nicétas à Constantinople et lui sauve la vie alors que la ville est mise à sac par les croisés latins. Il lui raconte alors sa vie depuis que son destin de jeune piémontais a croisé celui de Frédéric Barberousse, le célèbre empereur. Ce qui rend passionnant le récit de Baudolino, c'est que non seulement ce dernier est « un fieffé menteur » qui est tout le temps en train d'affabuler, mais c'est que ses propres mensonges deviennent des vérités ou donnent lieu à des événements historiques : Baudolino écrit des poèmes qui deviendront des chefs d'œuvre, invente des ouvrages ou des documents qui produiront des événements fondateurs : des armées seront levées, des villes entièrement détruites ou épargnées à cause de simples narrations élaborées par lui. Parmi ces histoires, celle $\mathrm{du}$ prêtre Jean sous-tend tout le roman et justifie même les dix derniers chapitres supplémentaires consacrées en apparence à l'expédition vers le royaume du prêtre Jean, mais qui constituent en réalité un amas inextricable de récits à tiroirs souvent abandonnés en cours.

3 Dans ce roman, Eco reconduit cette poétique baroque, saturée de cosmogonies, de réminiscences d'époques et de littératures diverses : cosmogonies médiévales, arabes, occidentales.

4 La couleur locale et autobiographique y est discrètement présente, que ce soit à travers la focalisation sur la ville d'Alexandrie et sur l'histoire de la région ou à travers le séjour de Baudolino à la Sorbonne, occasion pour l'auteur de distiller de nouveau quelques remarques ou pointes sur la France ou sur la Sorbonne. 
5 La religion est très présente dans le roman, plus précisément la question de la nécessité des reliques pour et par la foi. La majorité des personnages sont des prêtres, des dignitaires religieux ou des personnes s'autorisant des affiliations avec ses derniers. Tous recherchent le pouvoir à travers un seul type d'objets qui deviendra leur quête : les reliques religieuses existantes ou à inventer. Les lieux sont souvent des églises ou des cryptes. Les objets, des calices, des cadavres anonymes déclarés saints en vertu de la foi de qui les veut sanctifier, quand ce ne sont pas de simples ustensiles de la vie de tous les jours élevés au rang de relique. Exemple type, l'écuelle du père de Baudolino, devenue grâce à ce dernier le gradaal ou le graal. Ici Eco s'amuse avec les codes narratifs et culturels d'une gigantesque bibliothèque et nous invite, comme dans Le Nom de la rose, à rire ${ }^{1}$ de la vérité, mais au-delà du rire et du pastiche, le romancier se lance dans une entreprise délicate et sérieuse : comment raconter le mensonge, c'est-à-dire comment raconter la vérité ${ }^{2}$ ?

6 Il y a chez Eco une fascination - scientifique bien évidemment - pour le mensonge et pour le faux qui ne vient pas seulement de son activité de sémioticien, mais aussi d'une certaine prudence - je dirai de méthode - envers la vérité, parfois encombrante, souvent brève $e^{3}$, mais toujours nécessaire. C'est cette fascination pour le mensonge qui donne au personnage de Baudolino la force et le crédit nécessaire pour réussir sa carrière de menteur autorisé, pour ne pas dire admirable. Mais si Baudolino arrive à s'imposer comme un menteur admirable, ce n'est pas seulement grâce à un romancier rompu aux techniques de la fable et de la construction des personnages, c'est aussi parce que le romancier est assisté du sémioticien, qui lui insuffle tous les secrets sémiotiques du vrai et du faux. Ici l'auteur de Baudolino et l'auteur des Limites de l'interprétation, plus précisément des chapitres sur le vrai, le faux et la contrefaçon, réalise une complicité rarement égalée. L'objet de cette étude est de montrer l'étroite relation entre ces deux textes tout en analysant comment Eco s'y prend pour nous raconter la tragédie de la vérité et la farce du mensonge.

7 Pendant toute sa vie Baudolino n'a fait que mentir. Baudolino ne s'est jamais posé de questions sur les raisons qui le poussent à mentir. Ce n'est qu'en en rendant compte aux autres qu'il se crée l'occasion de répondre à cette question. Une des raisons évoquées par Baudolino lui-même est relative à une certaine impatience de voir se réaliser des choses, laquelle impatience n'est que le résultat d'une frustration devant ce qu'offre le monde. Cette fascination n'est pas propre à Baudolino, elle caractérise aussi les autres, comme il l'explique à Nicétas :

« Oui, mais il m'est toujours arrivé qu'à peine je disais j'ai vu ça, ou bien j'ai trouvé cette lettre qui dit comme ça (avec même la possibilité que je l'aie écrite moi), les autres donnaient l'impression qu'ils n'attendaient que cela. Tu sais, seigneur Nicétas, quand tu dis une chose que tu as imaginée, et que les autres te disent qu'il en est vraiment ainsi, tu finis par y croire toi-même ». (Eco 2000-2002 : 40)

8 La contagion de la vérité ou de la croyance est donnée à voir ici à travers la crédulité des autres qui finit par contaminer celui même qui en était l'origine. Cette situation ouvre les yeux de Baudolino sur une autre réalité : le prestige et le pouvoir de celui qui ment aux autres. Lorsqu'il visite Rome, Baudolino est déçu, car il ne retrouve rien des mirabilia que le prêtre lui a racontées sur cette ville. Sur le chemin du retour, il décida pourtant de raconter les mêmes mirabilia, d'en rajouter même et de découvrir l'effet de cela sur ceux qui l'écoutent : « ils étaient tous suspendus à mes lèvres » (Eco 2000-2002 : 46). 
9 Révéler des choses aux autres, même inventées, permet d'exercer une certaine influence. Cependant, il n'y a pas que le pouvoir d'en raconter à des personnes qui en semblent dépourvus qui fonde les mirabilia. Il y a aussi ce péché d'orgueil qui consiste à se prendre pour un démiurge. Face au même Nicétas, Baudolino tient à apporter cette précision sur ce qui le motive: «Je n'entends pas paraître humble. J'aime que des choses arrivent, dont je suis le seul à savoir qu'elles sont mon œuvre » (Eco 2000-2002 : 97). Dans d'autres contextes, Baudolino révèle les motifs du mensonge qui vont au-delà de l'orgueil pour embrasser des raisons existentielles :

« Voilà, seigneur Nicétas, dit Baudolino, lorsque je n'étais pas la proie des tentations de ce monde, je consacrais mes nuits à imaginer d'autres mondes. Un peu avec l'aide du vin, et un peu avec l'aide du miel vert. Il n'y a rien de mieux qu'imaginer d'autres mondes, dit-il, pour oublier combien est douloureux celui où nous vivons. Du moins, c'est ce que je pensais alors. Je n'avais pas encore compris que, à imaginer d'autres mondes, on finit par changer celui-ci ». (Eco 2000-2002 : 113).

10 En fait lorsque Baudolino revient sur les événements passés de sa vie, il ne montre pas seulement à Nicétas pourquoi il a contracté cette fâcheuse habitude de mentir et de mentir bien, il en profite aussi pour lui livrer de véritables techniques et trouvailles sur l'art de mentir. Ici, comme nous l'avions déjà évoqué, ce qui rend ces recettes intéressantes, c'est qu'elles s'appuient sur les propres recherches d'Umberto Eco, sémioticien sur le vrai, le faux et la contrefaçon. En effet dans Les Limites de l'interprétation, particulièrement dans l'examen de certaines notions comme celles du vrai et du faux, Eco donne à voir le malaise et les difficultés qui entourent leurs définitions ; il révèle aussi que, malgré ce manque de clarté et d'unité, ces notions sont nécessaires à l'homme. En ce qui concerne, par exemple, la contrefaçon, une sémiotique des mensonges permet de considérer « qu'on n'a pas affaire directement à des mensonges. Nous avons affaire plutôt à la possibilité de prendre un objet pour un autre, avec lequel il partage certains traits en commun " (Eco 1992: 179). Eco met ici l'accent sur l'aspect pragmatique du problème de la contrefaçon (l'intention de tromper), ce qui permet de tirer des conclusions susceptibles de mieux définir le faux : "Quelque chose n'est donc pas un faux à cause de ses propriétés internes, mais en vertu d'une identité prétendue. Ainsi les contrefaçons posent avant tout un problème pragmatique » (Eco 1992 : 183).

11 Donc le problème crucial d'une sémiotique du faux n'est pas de répertorier les erreurs du prétendant, mais de dire sur quoi le juge s'est appuyé pour démontrer que l'objet contrefait n'est pas authentique. Comme on le remarque, le problème est circulaire et accuse la fragilité de nos catégories apparemment les plus établies :

«La réflexion sur ces objets les plus contrefaits devrait nous dire en tout cas combien nos critères généraux pour l'identité sont hasardeux et combien des concepts tels que Vérité et Fausseté, Authentique et Faux, Identité et Différence, se définissent réciproquement et circulairement ». (Eco 1992 : 211)

12 La définition pragmatique d'Eco déplace la détermination du vrai et du faux du côté du destinataire ou du lecteur, en faisant peu de cas de l'objet et de ses qualités internes, tout en montrant la circularité du vrai et du faux. C'est ce critère de la foi comme critère objectif de l'authentification de l'objet qui fait dire à Nicétas : «De nombreuses reliques que l'on conserve ici à Constantinople sont de très douteuse origine, mais le fidèle qui les baise sent qu'il émane d'elles des arômes surnaturels. C'est la foi qui les rend vraies, non pas elles qui rendent vraie la foi » (Eco 2000-2002: 127). 
13 Il en va de même lorsqu'il s'agit de fabriquer un parfait faux. Umberto Eco montre dans Les Limites de l'interprétation combien il est facile de fabriquer un faux parfait pour ainsi dire en respectant les critères sur lesquels l'on s'appuie pour reconnaître un vrai. Un parfait faux du XVIème siècle par exemple est facile à faire, pourvu que l'on ait choisi un cadre et une toile d'époque sur laquelle on exécute une parfaite copie, etc. Bref, il s'agit de connaître parfaitement les critères à partir desquels on juge le vrai pour les reproduire dans la fabrication du parfait faux. Eco arrive à exposer de façon claire et convaincante comment les critères du vrai et du faux ne sont pas absolus et qu'ils sont parfois même fragiles, puisque faciles à reproduire.

14 C'est cette même règle que semble dicter le sémioticien au narrateur pour que les personnages l'appliquent dans l'invention de la lettre du prêtre Jean :

« Abdul réussit à soustraire nuitamment du scriptorium de l'abbaye de Saint-Victor un parchemin de grande valeur, jamais gratté. Il ne lui manquait qu'un sceau pour avoir l'air de la lettre d'un roi. Dans cette chambre, Baudolino, les yeux fermés, comme inspiré, dictait. Abdul écrivait car sa calligraphie, qu'il avait apprise dans les royaumes chrétiens d'Outremer, pouvait rappeler la façon dont écrit, en lettres latines, un Oriental ». (Eco 2000-2002: 152).

15 Ces quelques exemples nous suffisent à montrer la parfaite harmonie entre deux voix, celle du romancier et celle du sémioticien pour exprimer une même et seule fascination pour le mensonge et la vérité. L'auteur cependant ne se contente pas de rire de la vérité ni de dénoncer le mensonge, mais semble en défendre la nécessité. Eco livre dans ce roman l'esquisse d'une économie politique du mensonge et du mensonge religieux, qui se manifeste essentiellement par la relique. Le roman entier est traversé par cette question: Comment fabriquer des reliques? Tel est le programme qui absorbe Baudolino et ses compagnons dans la deuxième moitié du roman. Une fois maîtrisées ces quelques idées sémiotiques sur le faux et sur le vrai et une fois démontré, par la fabula, que la sémiotique est la science du mensonge, les personnages peuvent se lancer à fabriquer des reliques, mais ils découvrent qu'ils ne sont pas les seuls et qu'ils ont été précédés par d'autres personnages comme le noble dignitaire arménien nommé Ardzrouni qui avoue à Baudolino :

«Eh bien ce sont des reliquaires avec la vraie tête de saint Jean Baptiste. Chacun d'eux contient un crâne, traité avec des fumigations de façon qu'il rapetisse et paraisse très ancien. Je vis sur cette terre sans ressource aucune, point de champs à ensemencer et point de bétail, et mes richesses sont limitées. Je fabrique des reliques, c'est vrai, et elles sont très demandées aussi bien en Asie qu'en Europe ». (Eco 2000-2002: 324)

16 Il y a une nécessité politique de la relique, puisque des villes tombées pendant des siècles dans l'oubli et dans la stagnation peuvent du jour au lendemain devenir saintes et se transformer en véritables cités prospères.

17 Mais il importe de nuancer cette fascination pour le mensonge chez Baudolino et chez Eco. A priori Baudolino pourrait paraître comme un roman pessimiste et noir, car le personnage principal découvre qu'il peut mentir facilement et de façon efficace, que les autres aiment le mensonge, qu'il est très répandu et que les seules fois où il a essayé d'être sincère, il a produit une monstruosité ou a été lapidé; laquelle vision est soutenue par les recherches du sémioticien sur la circularité et sur les limites de notre univers mental, comme nous l'avions montré. Mais cela n'est que partiellement vrai, car Baudolino n'est ni cynique ni nihiliste ; à la fin du roman, il décide de rejoindre son Hypatie et de retrouver son monstre qu'il découvre aimer. Tout comme Eco lui-même 
qui, tout en admettant la circularité et la fragilité du vrai et du faux, ne cède pas à la tentation du nihilisme et de la béance, mais défend le sens de la limite et de la responsabilité.

\section{NOTES}

1. Voir ma communication au colloque de Versailles intitulée «le rire épistémique d'Umberto Eco ».

2. Voir un autre texte d'Eco écrit dans le même esprit: On the Truth a Fiction et repris dans Les Limites de l'interprétation.

3. Rappelons la petite phrase de Roberto dans l'Ile du jour d'avant : «...car la vérité est brève, le reste est commentaire ».

\section{ABSTRACTS}

Umberto Eco's fascination of lies is that of a semiotician for a human and semiotic phenomenon that lies at the crossroads of several issues relating to lies, narration and their close complicity that transcends our categories of thought as true and the false, the truth and the lie. Baudolino, the eponymous character of the novel, is a liar out of necessity to satisfy the thirst of men for stories that make people dream and trigger fears, hopes and obsessions. The purpose of this study is to examine the theme of lies in the novel, as well as the purely semiotic vision proposed by Umberto Eco.

La fascination d'Umberto Eco pour le mensonge est celle d'un sémioticien pour un phénomène humain et sémiotique qui se trouve au carrefour de plusieurs problématiques relatives au mensonge, à la narration et à leur étroite complicité qui transcende nos catégories de pensée comme le vrai et le faux, la vérité et le mensonge. Baudolino, personnage éponyme du roman, est menteur par nécessité pour satisfaire la soif des hommes pour les histoires qui font rêver et qui déclenchent les peurs, les espoirs et les hantises. L'objet de cette étude est d'examiner le thème du mensonge dans le roman, ainsi que la vision purement sémiotique qu'en propose Umberto Eco.

\section{INDEX}

Keywords: Baudolino, Eco, lie, truth, true, false, history

Mots-clés: Baudolino, Eco, mensonge, vérité, vrai, faux, histoire 
AUTHOR

MOHAMED BERNOUSSI

Université de Meknès 